\title{
The benefits and implementation challenges of the first state-wide comprehensive medication for addictions program in a unified jail and prison setting
}

\author{
Lauren Brinkley-Rubinstein $^{\mathrm{a}, \mathrm{b}, *}$, Meghan Peterson $^{\mathrm{c}}$, Jennifer Clarke ${ }^{\mathrm{d}}$, Alexandra Macmadu ${ }^{\mathrm{e}}$, \\ Ashley Truong ${ }^{\mathrm{c}}$, Kimberly Pognon ${ }^{\mathrm{c}}$, Morgan Parker ${ }^{\mathrm{a}, \mathrm{b}}$, Brandon D.L. Marshall ${ }^{\mathrm{e}}$, Traci Green ${ }^{\mathrm{f}}$, \\ Rosemarie Martin ${ }^{\mathrm{g}}$, Lynda Stein ${ }^{\mathrm{h}}$, Josiah D. Rich ${ }^{\mathrm{c}}$ \\ ${ }^{a}$ Department of Social Medicine, University of North Carolina at Chapel Hill, 333 S. Columbia Street, Chapel Hill, NC 27516, USA \\ ${ }^{\mathrm{b}}$ Center for Health Equity Research, University of North Carolina at Chapel Hill, 335 S. Columbia Street, Chapel Hill, NC 27514, USA \\ ${ }^{\mathrm{c}}$ Center for Prisoner Health and Human Rights, Miriam Hospital, 8 Third Street, Providence, RI 02906, USA \\ ${ }^{\mathrm{d}}$ Rhode Island Department of Corrections, 40 Howard Ave., Cranston, RI 02920, USA \\ e Department of Epidemiology, Brown University, 121 S. Main Street, Providence, RI 02903, USA \\ ${ }^{\mathrm{f}}$ Department of Emergency Medicine, Boston University Medical Center, Boston, MA 02118, USA \\ ${ }^{\mathrm{g}}$ Department of Behavioral and Social Science, Brown University, 121 S. Main Street, Providence, RI 02903, USA \\ ${ }^{\mathrm{h}}$ Department of Psychology, University of Rhode Island, 142 Flagg Road, Kingston, RI 02881, USA
}

A R T I C L E I N F O

Keywords:

Medications for addiction treatment

Incarceration

Overdose prevention

\begin{abstract}
A B S T R A C T
The prevalence of opioid use disorders among people who are incarcerated is high. People who are released from incarceration are at increased risk for overdose. The current study details the first year of implementation of a state-wide medications for addiction treatment (MAT) program in a unified jail and prison setting at the Rhode Island Department of Corrections in Cranston, Rhode Island. We conducted 40 semi-structured, qualitative interviews with people who were incarcerated and concurrently enrolled in the MAT program. Analysis employed a general, inductive approach in NVivo 12. We found that a majority of participants discussed program benefits such as reduced withdrawal symptoms, decreased prevalence of illicit drug use in the facility, improved general environment at the RIDOC, and increased post-release intentions to continue MAT. Suggested areas of improvement include reducing delays to first dose, increasing access to other recovery services in combination with MAT, improving staff training on stigma, and earlier access to medical discharge planning information prior to release. Our findings suggest that correctional MAT programs are acceptable to targeted populations and are a feasible intervention that may be transferable to other states.
\end{abstract}

\section{Introduction}

Opioid use disorder (OUD) and overdose have increased dramatically in the United States (US). From 1999 to 2014, drug overdose rates in the US nearly tripled (Rudd, 2016). Over the past two decades, nonmedical prescription opioid use contributed substantially to rising overdose rates (Calcaterra et al., 2013; Cerdá et al., 2013; Kenan et al., 2012). In more recent years, national initiatives to reduce opioid prescribing have produced modest declines in the number of prescription opioids dispensed (Dart et al., 2015). However, due in part to these supply-side interventions, from 2010 to 2014, the rate of heroin-involved overdose deaths in the US increased three-fold (Compton et al., 2016).
As people with OUD shift from the use of non-medical prescription opioids to heroin, the epidemic of opioid overdose has been exacerbated by contamination of the heroin supplies with illicitly-manufactured fentanyl and related compounds, as well as a shift to fentanyl use (Centers for Disease Control and Prevention, 2015). Several states have documented substantial increases in fentanyl-related overdose fatalities, including Rhode Island (RI) (Centers for Disease Control and Prevention, 2013; Lozier et al., 2015; Mercado-Crespo et al., 2014). In 2016, 58\% of all overdose deaths in RI involved fentanyl-an increase from $47 \%$ in 2015 and less than $5 \%$ in earlier years (Rhode Island Governor's Overdose Prevention and Intervention Task Force, 2015; Marshall et al., 2017; Truong et al., 2019).

Individuals with recent criminal justice (CJ) involvement have an

\footnotetext{
* Corresponding author at: University of North Carolina, 333 S. Columbia Street, MacNider Hall \#341, Chapel Hill, NC 27599, USA.

E-mail address: Lauren_Brinkley@med.unc.edu (L. Brinkley-Rubinstein).
} 
even greater increase in risk of overdose death during community reentry than people who have not been incarcerated as many people experience decreased tolerance to opioids after release (Binswanger et al., 2011, 2013; Binswanger et al., 2007; Farrell and Marsden, 2008; Merrall et al., 2010). In 2014 and 2015, 21\% of all fatal overdose victims in RI were incarcerated in the two years prior to death, an increase from 9\% in 2009 (Brinkley-Rubinstein et al., 2018a,b). In addition, in $2015,57 \%$ of overdose decedents who had recent criminal justice (CJ) involvement experienced a fentanyl-related overdose, an increase from 29\% in 2014 (Brink ley-Rubinstein et al., 2018a,b). In 2016, in response to the current opioid epidemic, the evolving fentanyl crisis, and the increased risk of overdose among those with recent CJinvolvement, the RI Department of Corrections (RIDOC) created the first-ever, statewide corrections-based comprehensive medications for addiction treatment (MAT) program that includes access to methadone, suboxone, and depot naltrexone combined with behavioral therapy. The decision of which medication to use is based upon the patient's needs and preferences.

Providing access to MAT in correctional settings can reduce postincarceration illicit opioid use (Connock et al., 2007; Mattick et al., 2009), criminal behavior (Kinlock et al., 2009), mortality and overdose risk (Deck et al., 2009; Degenhardt et al., 2011; Kerr et al., 2007), and HIV risk behaviors (MacArthur et al., 2012). Additional social, medical, and economic benefits to providing MAT to incarcerated persons with OUD are well-documented (Heimer et al., 2006; Mattick et al., 2009; McKenzie et al., 2012; Rich et al., 2015; Zaller et al., 2013; Dolan et al., 2003). The World Health Organization (World Health Organization, 2009) and the National Institute on Drug Abuse (National Institute on Drug Abuse, 2014) endorse the use of MAT to treat OUD in incarcerated populations, yet there has been little to no implementation or routinization of MAT in US jail and prison settings (Rich et al., 2015; Vestal, 2016). In RI, the comprehensive MAT program has been successful in reducing post-release overdose and has been cited as a national paradigm. A recent study by our group found that post-release overdose deaths decreased $60.5 \%$ among those with recent incarceration and induced a $12 \%$ decline in overall overdose deaths compared to the year before program implementation (Green et al., 2018). In an effort to build on these quantitative findings and provide a roadmap for other correctional entities who may be contemplating implementing MAT programs, the current study describes the perceived benefits and challenges encountered by participants in the RIDOC's MAT program.

\section{Methods}

We conducted 40 semi-structured, qualitative interviews with participants in the RIDOC MAT program. Interviews were conducted via the Evaluating the Implementation and Impact of a Novel Medication Assisted Treatment Program in a Unified Jail and Prison System (EMAT; R21DA044443) study. Inclusion criteria included current enrollment in the MAT program, being 18 years old and older, and being able to read and write in English. The MAT program has the following major components: 1) screening for opioid use disorder upon intake, 2) initiating or continuing suboxone, methadone, or naltrexone as clinically appropriate, 3) linking to community MAT upon release (via a medical discharge planner). In addition, all MAT program participants are required to attend regular group counseling sessions. The maximum dose for patients receiving methadone is $120 \mathrm{mg}$ unless ordered by a provider from the MAT service vendor; patients receiving buprenorphine have a maximum dose of $16 \mathrm{mg}$ unless determined by a provider that a higher dose is needed. Participants were recruited at the RIDOC during program group sessions by two research assistants trained in qualitative interviewing (MP, KP). During the program group sessions, the study was described, and participants were able to confidentially sign up for the study and be later contacted for an hour-long interview. Both interviewers (MP, KP) were female, had conducted research before in criminal justice settings, and were well versed in how best to build rapport and to work with people who are incarcerated.

The sample was purposively stratified to proportionally represent patients' type of MAT, time of MAT initiation (i.e., prior to vs. during incarceration), and facility of residence at the RIDOC (e.g., intake, minimum, and medium security).

All interviews were conducted in a private room without correctional officers present. The interviews were semi-structured and covered topics such as attitudes toward MAT, experiences in the MAT program, perceived benefits and challenges of the program, post-release substance use plans, and fentanyl perceptions. On average, the interviews were approximately one hour in length. Interviews were digitally recorded and later transcribed. All participants received a \$25 money order reimbursement for their time that was deposited into their commissary account. The study was approved by the Miriam Hospital's Institutional Review Board and the RIDOC Medical Research Advisory Group.

Qualitative data analysis employed a general inductive approach, which allows for research to be divided into codes and themes in line with the research objectives and the questions asked during interviews (Thomas, 2006). The coding team consisted of five individuals trained in qualitative research analysis (LBR, MP, KP, AT, AM) who utilized an initial codebook that mapped onto our study objectives. Four interviews were cross-coded by all five team members to further refine and add to the codebook, ensure coder agreement and uniform use of the codes. After this initial coding exercise, all codes were compiled into a final codebook, and three members of the coding team (MP, KP, and AT) coded the remaining transcripts. Final analyses were performed in NVivo 12.

\section{Results}

\subsection{Participant characteristics}

Participants ranged from 22 to 66 years old with a mean age of 37.2 . Of those participants, $50 \%(n=20)$ were receiving methadone, $47.5 \%$ $(\mathrm{n}=19)$ were receiving buprenorphine, and one person $(2.5 \%)$ was receiving depot naltrexone (this breakdown is proportional to the number of patients at the RIDOC receiving each medication). In total, $50 \%$ of participants had started their current MAT prescription in the community prior to arrest and 50\% had initiated their current MAT prescription while they were incarcerated at the RIDOC. Most participants were male $(70 \% ; \mathrm{n}=28)$ and White $(82.5 \% ; \mathrm{n}=33)$. Five percent $(\mathrm{n}=2)$ were Black, $12.5 \%(\mathrm{n}=7)$ identified as belonging to an "other" racial group, and $10 \%(\mathrm{n}=4)$ were Hispanic. Participants also predominantly identified as heterosexual $(87.5 \%, \mathrm{n}=35)$ with $(5 \%$; $\mathrm{n}=2)$ identifying as gay and $7.5 \%(\mathrm{n}=3)$ identifying as bisexual. Overall, $40 \%(n=16)$ had finished high school, $20 \%(n=8)$ had not completed high school, and the remainder reported completing education beyond a high school degree $(40 \%, n=16)$. A majority of participants reported that before incarceration, they had been using heroin (95\%; $\mathrm{n}=38$ ); thirty $(75 \% ; \mathrm{n}=30$ ) used prescription opioids nonmedically, 21 (53\%) used cannabis, $12(30 \%)$ reported non-medical benzodiazepine use, and 8 (20\%) reported having used alcohol when asked an open-ended question to describe their substance use history in general.

\subsection{MAT program benefits}

In general, participants' reported experiences with the program were very positive. A majority discussed benefits, such as not having to go through withdrawal, but many also discussed unexpected benefits, including decreased prevalence of illicit drugs inside RIDOC, improved general environment at the RIDOC, and increased post-release intentions to continue MAT. 


\subsubsection{Incarceration without withdrawal}

Most participants stated that avoidance of withdrawal was a major benefit of the MAT program at the RIDOC. As one 36-year-old male participant said after being asked about experiences in the program: "[The program] definitely helped me to like, you know, just regulate and get back on the path of, you know, not being sick every day." The participant reported that, relative to prior incarcerations, his experience of incarceration had improved without withdrawal symptoms. Another 33-year-old male participant compared his current experience to a previous time he had been incarcerated and lacked access to MAT, noting that he felt a reduction in withdrawal symptoms:

"It's helped me big time because last time I was here I struggled almost half my [sentence]. I was sick as a dog. I just felt awful. Now, this time around, I don't have to be like that. I can be normal. I don't have to feel sick and feel like I'm half dead. So, I think it's big. I think they're doing good offering that in the prison system now, finally. I think they should do it in other states."

Other participants echoed this sentiment and discussed the wide reach of the program given that many people in the RIDOC were incarcerated due to crimes related to their substance use disorder. One participant, for example, discussed how the program had helped ease his withdrawal symptoms. This participant perceived that the program was intended both to help people with substance use disorders and to reduce recidivism among the broader population. When asked how the participant would feel without the program in place, the 36-year-old male participant responded:

"Yeah, I'd be sick. I'd be uncomfortable, and my mind would be all over the place. It's 100 percent better. You're just making things a lot easier for people. I mean this is jail, don't get me wrong, but this [is] medication that doctors give us, you know? So, it's like, you guys are doing the right thing. [...] Because we're trying to get our lives back together. You know people that are on the stuff. So, with taking that away and shooting guys back to the streets just to put them back in the same position, and it's really not the cure for that. They're usually here because of substances. Ninety percent, well, I don't know the percentage, but most of these cats are here from stealing or whatever they're doing, you know. So, you're helping them out."

The participant, therefore, explained that he felt that the program would reduce recidivism and address the social circumstances surrounding addiction that led to incarceration in the first place. The participant noted that MAT was merely a medical treatment to a health condition and would contribute to a reduction in incarceration over time.

\subsubsection{Decreased illicit substances at the RIDOC}

Many participants stated that an ancillary benefit to the program was a lower prevalence of illicit drugs in the facility due to decreased need to use among people who are incarcerated. When asked how the environment at the facility would be different without the MAT program, one 22-year-old male participant said: "There'd be more drugs. There'd be a lot more drugs floating around jail, I think. A lot more. We're addicts. We're going to get high. If we want to get high, we're going to get high". The participant perceived that the MAT program had contributed to a reduction in substance use in the facility, as people experiencing opioid use disorder would try to curb withdrawal regardless of whether withdrawal management was allowed. Another 41year-old male participant similarly stated that his need to use illicit drugs was no longer an issue:

"Unfortunately, there is drugs in the prison systems and being on this [MAT], that is not even on my radar. I don't have to worry about getting something off somebody and doing that whole criminal activity or owing people anything. I do my own program in here, and
I'm good. I don't need anything else. It's a blessing. It's a blessing that finally people are realizing isn't the devil."

Relatedly, another 55-year-old male participant stated that because of the MAT program, there were fewer overdoses from illicit drugs among incarcerated people while at RIDOC: "[If there was no MAT program] there'd be more overdoses, and people would be dropping like flies because of the potency of the heroin in the street now with fentanyl. And they would bring it in the system, and people would be dropping. It would be more people in caskets than anything else."

\subsubsection{Improved facility environment}

While discussing the benefits of the MAT program, participants often discussed a general improvement in the environment at the RIDOC. For instance, one participant highlighted that fewer people would be tempted to use illicit substances during incarceration, which could lead to less violence. When asked to imagine that there was no MAT program in place, the 22-year-old male participant reported that conditions in the RIDOC would feel more unsafe:

"If there was no MAT program, it'd be more drugs in the prison, and with more drugs in the prison, anything is liable to happen. When people get high, they're not themselves. I know when I get high, I'm not myself. I'm actually really mean and like really angry all the time. So, I mean it can be a lot less safe and probably be more fights, more violence".

Another participant speculated that the improvement in the facility environment would extend to non-MAT program participants. While discussing the benefits of the program, the 36-year-old male reported:

"When they come in, and [people with OUD are] sick, they're sick. They definitely need methadone, and I think it's great that people are sick, and they come in here and they can get on something that helps them. That's awesome. [People] over there puking on themselves, you know what I mean, and like some people don't do drugs - they're disgusted. Like, you're disgusting, you know I mean? We don't want to come into jail and then live with somebody who's over there puking. You know what I mean? It's better for the people that aren't on drugs, and it's better for the people who are on drugs. It's better for everybody because nobody's sick, nobody's puking themselves. I've been there, you know?"

Relatedly, some participants reported that the MAT program had eased the burden on staff. For example, one 41-year-old male participant who was asked about how RIDOC nurses perceived the program stated:

"I think they understand what the potential could be and is. It's less work for them having people not come in and being sick all the time. They understand how much of a mental thing that is. You almost do anything to not feel that way. You'll lie. I had a roommate that faked having a seizure, a stroke just to get his meds to feel better. I think they're a little more sympathetic [after MAT program implementation]."

This participant reported that other people who were incarcerated typically needed less care than people who were experiencing withdrawal symptoms. From this participant's perspective, reducing the number of people who were experiencing withdrawal symptoms created a more balanced workload for RIDOC staff. Participants therefore highlighted how the environmental changes at the facility extended beyond individual participants to other people who were incarcerated and staff.

\subsubsection{Changed post-release substance use intentions}

In addition to having positive implications during incarceration, participants also discussed the personal impact of the MAT program on post-release plans. Specifically, they focused on how access to MAT at 
RIDOC would improve their ability to discontinue use of illicit substances during community re-entry. When asked how the program had been beneficial, one 31-year-old male participant reported:

"I mean I think, like I said, I think it's a godsend. I mean I think, you know, I think they should have this in other prisons especially because this is the time where a lot of people make the decision on if they're going to stay clean or not. Because you gather a little time under your belt and then you say all right, well, you know, maybe I will stay clean. And then, well, how am I going to do it? I've got, you know. Or if I get out, I'll use. You have a little time to step back and look at things, see the way things are for actually for what they are. You know? Without a clouded distorted view of that, you know, with drug fueled way that you're living out there."

The participant reported that receiving MAT while at the RIDOC allowed him to think about abstaining from future substance use. $\mathrm{He}$ noted that his attitude had changed toward reducing substance use over the period of time that he had been incarcerated and enrolled in treatment. Another 36-year-old male participant highlighted how access to MAT while incarcerated meant that he would not experience cravings post-release and therefore abated the need to return to illicit substances:

"Well, I just know that you can get on the program here and stay on it through your bid, and when you get out, you're hooked up, and you don't have to run to the street, you know. A lot of guys are coming in, they'd be sick. As soon as they get out, they run right out and get a bag of dope, and that's it".

This sentiment was echoed by the majority of participants who did not intend to use opioids and other substances post-release; these participants instead expressed intention to continue on MAT treatment post-release through care linkage.

\subsection{MAT program areas for improvement}

Most participants expressed satisfaction with the program, but many also had suggestions for optimal program delivery. Suggested areas of improvement centered on four key themes: reducing delays to first dose, increasing access to other recovery services in combination with MAT, increasing [or "improving"] staff training on stigma, and earlier access to medical discharge planning information prior to release.

\subsubsection{First dose delays}

The majority of participants did not report delays to accessing MAT during their incarceration; however, it was an issue for a few participants. While most participants explained that they had received their doses in a timely matter, some participants cited delays in receiving MAT. They discussed how delays undercut the therapeutic benefits of MAT that would have minimized their withdrawal symptoms. A 35year-old male participant who had not been on MAT in the community before his incarceration also cited delays after specifically requesting MAT to aid in withdrawal:

"The first day upon intake I told [a nurse] that I was about to be sick and I needed to speak to someone about getting on methadone because I came in on nothing. And she said she will let the nurse know and do I fill out a slip. [...] I waited for a few days to get called. I didn't get called. I sent in a slip, and I ended up getting called down [...] To interview me at first, and then I saw a doctor a few days later. The reason it took so long, I guess, is there are lockdowns of certain things in the jail system that I couldn't go around anything today. [...] It was around a seven, eight, nine - seven to nine-day process, where I felt like shit the whole time, but I still had to do it. No sleep."

For participants who were continuing on a MAT prescription that originated in the community, some experienced delays based on inability to report information such as the name of a prescribing doctor from the past. One 34-year-old participant, for example, noted that he could only give a vague description of the previous doctor that prescribed him MAT.

"What they did was they said, 'Oh, since you were on it before it will be quicker for you to get it' so I told them I was on it. I didn't know the name of the doctor; I just said, 'Hey, yeah, I was on it.' I told them about where it was and the doctor I went to so he said, 'Oh, I know where that is.' He couldn't get the doctor to release any information so because of that they made it look like I was lying and they didn't give me anything. He said, 'Oh, well, we can't prove you were on it so that doctor is not going to give it to you.' [...] That's why it took four months to get on it."

The participant continued to try to obtain MAT and stated that he was finally prescribed it after detailing to staff how he had overdosed four times in the community. The process of coordinating information between community providers and the RIDOC sometimes impacted ability to quickly uptake MAT.

\subsubsection{Access to recovery strategies in combination with MAT often requested}

When asked what participants would improve about the MAT program, many stated that access to other recovery strategies in combination with MAT would be beneficial. One 31-year-old male participant noted that he would prefer more frequent and a diverse range of programs:

"I mean they could have like - they can have more meetings here. I mean they have only one AA Thursday; every AA [is] Thursday once a week here. And I never heard anything about NA here to be honest with you. I mean they could have, like, at least a couple of meetings a week. I mean they only have one."

He continued that he would also benefit from more one-on-one services: "Well, I just think it would be better off if it was like - I would prefer something more one on one. Like, obviously, they couldn't do something in such a large place with so many addicts. You know what I mean? But at least get a little bit deeper because most addicts aren't afraid to talk about it." Similarly, another 35-year-old male participant stated the need for either individualized counseling or structured group meetings: "I mean counseling is good for anybody. Problems or no problems. It's a nice sounding board. I mean sometimes you just need to say something out loud to make it click. I think if we had individual counseling and a structured group in which we actually cover things, like, there's no workbook. If there was a workbook, I think that would work out great for everything, you know".

\subsubsection{Staff education and training}

Many participants stated that there was a need for more staff education and training about MAT, the benefits of MAT, and about addiction in general. One 41-year-old male participant said specifically about correctional officers:

"You know, [the correctional officers] think it's like free drugs. They don't know. They're uneducated about what it actually is and what it does. They think that some of these dudes are just on it to get high. They don't understand that once you get used to it, you don't get anything really off of it."

Another 52-year-old female participant relayed a similar concern and underscored the importance of educating correctional officers about the extended benefits of MAT:

“They [correctional officers] don't believe in it. They're not drug addicts so they don't understand it for one. If somebody could counsel - they should have a meeting for them to understand why we're on it because no one explained to them. They just think well, 
we're criminals [...] There's a number of them that feel that way. So, if they was to have counseling or groups that they can sit and learn why it's beneficial to the people that take it so we don't go out and die or prevent us from stealing to get drunk. I think it would help the crime rate and help the death rate, the stealing anyway."

Another participant discussed how some medical staff weren't in favor of the program: "I could count - I know two of them that are totally against it, could not care less if you take it or not, but for the majority, they understand. There's a couple that don't understand it and don't understand why they're helping us with it because they don't get it. They're not in the drug programs. A little more schooling on it for them would be better, I think."

Many participants noted that they felt stigma from nurses and correctional officers, and when pressed further, explained that they believed that stigma stemmed from a lack of understanding. After explaining that they felt looked down upon by nurses and COs, the 26year-old male stated: "You see the problem is that I don't - it's a lot of people don't really understand how addiction is. They think it's just they think that we use just 'cause we want to get high or we just want to use. They just don't understand how the addiction really works."

\subsubsection{More information wanted about linkage before discharge}

Participants expressed a desire to learn more about the linkage process to community-based MAT before discharge. Additionally, participants stated that they would prefer to learn about the linkage to care process before their scheduled meeting with a discharge planner, which often occurs shortly before release. A 35-year-old male participant stated:

"I'm really nervous about the leaving part, to be honest. I get my dose today, but I get out of here tomorrow. I'm like, you know what I mean? Like, where am I going to get my dose? The discharge part because I haven't gone through it, and I don't know how it's going to go; and [the MAT provider who dispenses MAT at RIDOC] only has so many places where you can go and dose. [The MAT provider who dispenses MAT at RIDOC] can only do so much to help you get set up. I mean it's not like they're going to hold your hand once you walk out of here, you know. In my shoes, I'm being told that because of security issues and what not, I'll know $48 \mathrm{~h}$ in advance before I'm discharged when I make parole. That's even like the people who I work with. Well, they know maybe $48 \mathrm{~h}$ in advance before I'm discharged. They say it's a security issue. Whatever. [...]That's just the way it is, and while I'm not worried about, you know, going into full withdrawal in that $48 \mathrm{~h}$."

This participant expressed anxiety about uncertainty post-release and thought that knowing well in advance that he had a plan postrelease would ease his worries. Another 30-year-old female participant discussed how because she had been recently incarcerated and was not likely to stay in the facility for a long time, she had not received as many resources as those with longer sentences. She discussed how the process would be simplified if she was provided with resources to plan re-entry herself:

Interviewer: Okay. So you have an appointment with the discharge planner. Why do you think that no one has talked to you about the program yet?

Respondent: Cause I just got here Friday. [...] And I leave in thirty days and they're kind of like eh, you know, because a lot of girls are like going to be here longer.

[...]

Interviewer: So ideally for like people like you who are staying for a very short period of time and then being released, what kind of like attention would you have?

[...]

Respondent: You know it would be cool if like you could have a phone, right, or like there would be a phone that you could use for just programs or like discharge planner or something like that. [...] Like if I could do it on my own. [...] Do you know what I mean? That would be helpful.

Interviewer: So like a hotline or something or more hot topics? Respondent: Or just like more like paperwork with numbers on it or something where I could like call my family and be like hey. Like here are the numbers. Can you help me get into it? Because if they can't do it for you like you have to have a way to do it yourself like. [...] I'm sure they could get like a packet with like numbers or something like.

Another participant stated that he had five weeks left in the facility before anticipated release. While no one had discussed discharge planning with him yet, he felt that it was likely that someone would contact him for an appointment with a discharge planner before his release. Asked about challenges to continuing MAT, the 34-year-old male stated:

"If nobody talks to me before I leave and shows me my options of where to go when I get out, that's something I'm definitely going to need, so that might make it difficult. If there is nobody to talk to and I have to figure it out when I get out - I mean it's going to make it difficult. I'll figure it out, but it would be nice because I know somebody is in here that can tell me where to go, whether it's an inmate or a counselor or somebody. There is a list of places I can go. I know there is, so I'm hoping I can get it before I leave."

\section{Discussion}

Our study documents participants' mostly positive experiences with and attitudes toward the MAT program at the RIDOC, with a major benefit being withdrawal symptom management. Ancillary benefits of the MAT program included a decreased supply and demand for illicit drugs at the RIDOC, which contributed, in part, to an improvement in the RIDOC environment overall. Participants also viewed the program as positively influencing their post-release substance use intentions, with many individuals reporting a desire to continue MAT post-release. This qualitative evaluation of the first statewide program of its kind that provides access to all three FDA approved MAT options provides insight into how best to optimize future program implementation at the RIDOC and other correctional sites.

Despite benefits of the MAT program, participants also noted room for improvement. Challenges reported by participants included first dose delays largely related to lapses in communication between community treatment providers and the RIDOC. This delay in treatment often led to withdrawal symptoms that reduced potential therapeutic benefits of MAT. Additionally, participants emphasized preferences for additional recovery resources while incarcerated and expressed a need for proactive communication about the medical discharge process postrelease. Many participants expressed the desire to continue MAT in the community, but this absence of support inhibited their ability to adequately prepare for treatment continuation following release from incarceration. Participants also stressed the need for more training and education for nurses and correctional officers.

Previous studies have found similar findings relating to access to MAT while incarcerated and reduced substance use after incarceration. We previously demonstrated that prisoners who had access to methadone pre-release were seven times more likely than their untreated counterparts to seek treatment at a community methadone clinic within 30 days post-release (Rich et al., 2015). Long term outcomes from this same study showed that individuals who had access to methadone during incarceration were more likely to be engaged in continuous MAT treatment one-year post-release (Brinkley-Rubinstein et al., 2018a, b). Additionally, a study comparing buprenorphine treatment initiation before and after release revealed a difference in the number of days that participants continued treatment. Participants who began treatment 
pre-release continued buprenorphine on average 44.1 days longer than those who started taking the medication after release (Gordon et al., 2017) Although this study did not demonstrate a difference in self-reported illicit opioid use among the in-prison treatment and post-release treatment groups after 12 months, (Gordon et al., 2017) Kinlock et al. did find a significant difference in ill icit opioid use 12 mon ths postrelease among persons receiving methadone $(48.7 \%$ among the inprison treatment group vs. $25 \%$ in the post-release treatment group, respectively) (Kinlock et al., 2009). The discrepancy in results between buprenorphine and methadone treatment programs is particularly relevant to the current RIDOC MAT program, as participants received one of three treatments: buprenorphine, methadone, or depot naltrexone. Potential differences in program adherence post-release should be investigated in future studies.

Participants' positive experiences and perceived benefits from the program suggest the feasibility of implementing a comprehensive MAT program in correctional settings. Our findings also underscore the ancillary benefits of MAT access, including less need for illicit substances during incarceration and improved facility environment for program participants, other incarcerated people, and staff. Highlighting these types of benefits could be useful in early discussions about whether or not to implement corrections-based MAT programs or in staff education during early implementation of new programs and could help offset concerns for other concerns cited by correctional officers re lated to diversion of suboxone. This information could also help diminish stigma, an issue raised by participants in the current study, by helping individuals understand the widespread collateral benefits of providing MAT during incarceration. Participants also noted a lack of other support services complementing MAT, so development and implementation of these resources could be further explored. The Connecticut Offender R e-entry P rogram ( CORP) i ncludes i ndividualized re-entry planning that links the incarcerated to community health resources (Smith-Merry et al., 2019). The program also proactively places those at risk of homelessness on waiting lists while incarcerated to improve access to mental health services post-release. This proactive approach led to a reduction in recidivism rates. MAT programs can utilize this model as a framework to increase access to other support services before and after incarceration.

\subsection{Conclusion}

In 2016, the RIDOC became the first statewide system to implement a comprehensive MAT program that offers all three FDA approved MAT options. Post-program implementation overdoses among those previously incarcerated at the state-level decreased. In the current paper, we present qualitative findings relevant to participants experience with the MAT program. Participants expressed broad satisfaction with program, unanticipated ancillary benefits s uch a s i mproved f acility environment, and some room for improvement. These findings $\mathrm{c}$ an be used by other correctional facilities that are interested in implementing MAT programs as talking points relevant to the benefits of MAT programs and in anticipation of various implementation challenges.

\section{Role of funding source}

Funding for this project includes the National Institute of Drug Abuse (R21DA043487) and the John and Laura Arnold Foundation.

\section{Contributors}

LBR: Conceptualized the paper and took the lead on the paper. She also led the project and supervised the development of the interview protocol, interview process, and data analysis.

MePe: Contributed to the writing of the paper, conducted interviews, and aided in data analysis.

JC: Contributed to the writing and review of the paper.
AM: Contributed to the writing of the paper and data analysis AT: Contributed to the writing of the paper and data analysis. KP: Contributed to the writing, conducted interviews, aided in data analysis.

MoPa: Contributed to the writing of the paper.

BM: Contributed to the writing of the paper.

TG: Contributed to the writing of the paper.

RM: Contributed to the writing of the paper.

LS: Contributed to the writing of the paper.

JR: Contributed to the writing of the paper and is the PI of the project.

All authors have read and approved the final manuscript.

\section{Declaration of Competing Interest}

The authors have no conflict of interest.

\section{References}

Binswanger, I.A., Stern, M.F., Deyo, R.A., Heagerty, P.J., Cheadle, A., Elmore, J.G., Koepsell, T.D., 2007. Release from prison-a high risk of death for former inmates. N. Engl. J. Med. 356 (2), 157-165.

Binswanger, I.A., Blatchford, P.J., Lindsay, R.G., Stern, M.F., 2011. Risk factors for allcause, overdose and early deaths after release from prison in Washington state. Drug Alcohol Depend. 117 (1), 1-6.

Binswanger, I.A., Blatchford, P.J., Mueller, S.R., Stern, M.F., 2013. Mortality after prison release: opioid overdose and other causes of death, risk factors, and time trends from 1999 to 2009. Ann. Intern. Med. 159 (9), 592-600.

Brinkley-Rubinstein, L., Macmadu, A., Marshall, B.D., McKenzie, M., Rich, J.D., Green, T.C., 2018a. Increased risk of fatal overdose associated with recent criminal justice system involvement: findings from a recent outbreak of illicit synthetic fentanyl overdose. Drug Alcohol Depend. 185, 189-191.

Brinkley-Rubinstein, L., McKenzie, M., Macmadu, A., Larney, S., Zaller, N., Dauria, E., Rich, J., 2018b. A randomized, open label trial of methadone continuation versus forced withdrawal in a combined US prison and jail: findings at 12 months postrelease. Drug Alcohol Depend. 184, 57-63.

Calcaterra, S., Glanz, J., Binswanger, I.A., 2013. National trends in pharmaceutical opioid related overdose deaths compared to other substance related overdose deaths: 1999-2009. Drug Alcohol Depend. 131 (3), 263-270.

Cerdá, M., Ransome, Y., Keyes, K.M., Koenen, K.C., Tracy, M., Tardiff, K.J., Vlahov, D., Galea, S., 2013. Prescription opioid mortality trends in New York City, 1990-2006: examining the emergence of an epidemic. Drug Alcohol Depend. 132 (1-2), 53-62.

Centers for Disease Control and Prevention, 2013. Notes from the field: acetyl fentanyl overdose fatalities-Rhode Island, March-May 2013. MMWR Morb. Mortal. Wkly. Rep. 62 (34), 703.

Centers for Disease Control and Prevention, 2015. CDC Health Advisory: Increases in Fentanyl Drug Confiscations and Fentanyl-Related Overdose Fatalities. HAN Health Advisory.

Compton, W.M., Jones, C.M., Baldwin, G.T., 2016. Relationship between nonmedical prescription-opioid use and heroin use. N. Engl. J. Med. 374 (2), 154-163.

Connock, M., Juarez-Garcia, A., Jowett, S., Frew, E., Liu, Z., Taylor, R.J., Fry-Smith, A., Day, E., Lintzeris, N., Roberts, T., Burls, A., 2007. Methadone and buprenorphine for the management of opioid dependence: a systematic review and economic evaluation. NIHR Health Technol. Assess. Program. Exec. Summ. (NIHR J. Lib.) 11 (9), $1-171$.

Dart, R.C., Surratt, H.L., Cicero, T.J., Parrino, M.W., Severtson, S.G., Bucher-Bartelson, B., Green, J.L., 2015. Trends in opioid analgesic abuse and mortality in the United States. N. Engl. J. Med. 372 (3), 241-248.

Deck, D., Wiitala, W., McFarland, B., Campbell, K., Mullooly, J., Krupski, A., McCarty, D. 2009. Medicaid coverage, methadone maintenance, and felony arrests: outcomes of opiate treatment in two states. J. Addict. Dis. 28 (2), 89-102.

Degenhardt, L., Bucello, C., Mathers, B., Briegleb, C., Ali, H., Hickman, M., McLaren, J., 2011. Mortality among regular or dependent users of heroin and other opioids: a systematic review and meta-analysis of cohort studies. Addiction 106 (1), 32-51.

Dolan, K.A., Shearer, J., MacDonald, M., Mattick, R.P., Hall, W., Wodak, A.D., 2003. A randomised controlled trial of methadone maintenance treatment versus wait list control in an Australian prison system. Drug Alcohol Depend. 72 (1), 59-65.

Farrell, M., Marsden, J., 2008. Acute risk of drug-related death among newly released prisoners in England and Wales. Addiction 103 (2), 251-255.

Green, T.C., Clarke, J., Brinkley-Rubinstein, L., Marshall, B.D., Alexander-Scott, N., Boss, R., Rich, J.D., 2018. Postincarceration fatal overdoses after implementing medications for addiction treatment in a statewide correctional system. JAMA Psychiatry 75 (4), 405-407.

Gordon, M.S., Kinlock, T.W., Schwartz, R.P., O'Grady, K.E., Fitzgerald, T.T., Vocci, F.J., 2017. A randomized clinical trial of buprenorphine for prisoners: findings at 12 months post-release. Drug Alcohol Depend. 172, 34-42.

Heimer, R., Catania, H., Newman, R.G., Zambrano, J., Brunet, A., Ortiz, A.M., 2006. Methadone maintenance in prison: evaluation of a pilot program in Puerto Rico. Drug Alcohol Depend. 83 (2), 122-129.

Kenan, K., Mack, K., Paulozzi, L., 2012. Trends in prescriptions for oxycodone and other 
commonly used opioids in the United States, 2000-2010. Open Med. 6 (2), e41.

Kerr, T., Fairbairn, N., Tyndall, M., Marsh, D., Li, K., Montaner, J., Wood, E., 2007 Predictors of non-fatal overdose among a cohort of polysubstance-using injection drug users. Drug Alcohol Depend. 87 (1), 39-45.

Kinlock, T.W., Gordon, M.S., Schwartz, R.P., Fitzgerald, T.T., O’Grady, K.E., 2009. A randomized clinical trial of methadone maintenance for prisoners: results at 12 months postrelease. J. Subst. Abuse Treat. 37 (3), 277-285.

Lozier, M.J., Boyd, M., Stanley, C., Ogilvie, L., King, E., Martin, C., Lewis, L., 2015. Acetyl fentanyl, a novel fentanyl analog, causes 14 overdose deaths in Rhode Island, March-May 2013. J. Med. Toxicol. 11 (2), 208-217.

MacArthur, G.J., Minozzi, S., Martin, N., Vickerman, P., Deren, S., Bruneau, J., Degenhardt, L., Hickman, M., 2012. Opiate substitution treatment and HIV transmission in people who inject drugs: systematic review and meta-analysis. BMJ 345, e5945.

Marshall, B.D., Krieger, M.S., Yedinak, J.L., Ogera, P., Banerjee, P., Alexander-Scott, N.E., Rich, J.D., Green, T.C., 2017. Epidemiology of fentanyl-involved drug overdose deaths: a geospatial retrospective study in Rhode Island, USA. Int. J. Drug Policy 46, $130-135$.

McKenzie, M., Zaller, N., Dickman, S.L., Green, T.C., Parihk, A., Friedmann, P.D., Rich, J.D., 2012. A randomized trial of methadone initiation prior to release from incarceration. Subst. Abuse 33 (1), 19-29.

Merrall, E.L., Kariminia, A., Binswanger, I.A., Hobbs, M.S., Farrell, M., Marsden, J., Hutchinson, S.J., Bird, S.M., 2010. Meta-analysis of drug-related deaths soon after release from prison. Addiction 105 (9), 1545-1554.

Mercado-Crespo, M.C., Sumner, S.A., Spelke, M.B., Sugerman, D.E., Stanley, C., 2014. Notes from the field: increase in fentanyl-related overdose deaths-Rhode Island, November 2013-March 2014. MMWR Morb. Mortal. Wkly. Rep. 63 (24), 531.

Mattick, R.P., Breen, C., Kimber, J., Davoli, M., 2009. Methadone maintenance therapy versus no opioid replacement therapy for opioid dependence. Cochrane Database Syst. Rev.(3).
National Institute on Drug Abuse, 2014. Principles of Drug Abuse Treatment for Criminal Justice Populations: A Research Based Guide. National Institutes of Health, National Institute on Drug Abuse. NIH publication No. 11-5316, Bethesda, MD.

Rich, J.D., McKenzie, M., Larney, S., Wong, J.B., Tran, L., Clarke, J., Noska, A., Reddy, M., Zaller, N., 2015. Methadone continuation versus forced withdrawal on incarceration in a combined US prison and jail: a randomised, open-label trial. Lancet 386 (9991), 350-359.

Rhode Island Governor's Overdose Prevention and Intervention Task Force, 2015. Rhode Island's Strategic Plan on Addiction and Overdose: Four Strategies to Alter the Course of an Epidemic. Rhode Island Department of Health.

Rudd, R.A., 2016. Increases in drug and opioid-involved overdose deaths-United States, 2010-2015. MMWR Morb. Mortal. Wkly. Rep. 65.

Truong A.Q., Macmadu A., Peterson M., Brinkley-Rubinstein L., Potter N., Green T.C., Clarke J., Rich J.D. Fentanyl-related overdoses during incarceration: a comprehensive review. Submitted to Public Health Rep.

Smith-Merry, J., Mellifont, D., McKenzie, K., Clenaghan, P., 2019. A narrative review of mental health support for people during transition from incarceration to community: the grass can be greener on the other side of the fence. J. Ment. Health 28 (2), 189-197.

Thomas, D.R., 2006. A general inductive approach for analyzing qualitative evaluation data. Am. J. Eval. 27 (2), 237-246.

Vestal, C., 2016. At Rikers Island, A Legacy of Medication-Assisted Opioid Treatment. The Pew Charitable Trusts, Stateline.

World Health Organization Dept. of Mental Health Substance Abuse WHO, International Narcotics Control Board UNOoDaC, 2009. Guidelines for the Psychosocially Assisted Pharmacological Treatment of Opioid Dependence. World Health Organization.

Zaller, N., McKenzie, M., Friedmann, P.D., Green, T.C., McGowan, S., Rich, J.D., 2013. Initiation of buprenorphine during incarceration and retention in treatment upon release. J. Subst. Abuse Treat. 45 (2), 222-226. 